\title{
Nucleus Detection of Uterine Cervical Pap-Smears using Contour Trucking Method and Fuzzy Reasoning Rule
}

\author{
Hyunjun Woo ${ }^{1}$, Young Woon Woo ${ }^{2}$ and Kwang-Baek Kim ${ }^{3}$ \\ ${ }^{1}$ College of Medicine, Seoul National University, Seoul, Korea \\ 2 Dept. of Multimedia Eng., Dong-Eui University, Busan, Korea \\ ${ }^{3}$ Dept. of Computer Eng., Silla University, Busan, Korea \\ junwoo1207@snu.ac.kr,ywwoo@deu.ac.kr,gbkim@silla.ac.kr
}

\begin{abstract}
In this paper, we apply a set of algorithms to classify normal and cancer nucleus from uterine cervical pap-smear images. First, we use lightening compensation algorithm to restore color images that have defamation through the process of obtaining $400 x$ microscope magnification. Then, we remove the background from images with the histogram distributions of $R G B$ regions. We extract nucleus areas from candidates by applying histogram brightness, Kapur method, and our own 8-direction contour tracing algorithm. Various binarization methods, cumulative entropy, masking algorithms are used in that process. Then, we are able to recognize normal and cancer nucleus from those areas by using three morphological features - directional information, the size of nucleus, and area ratio - with fuzzy membership functions and deciding rules we devised. The experimental result shows our method has low false recognition rate.
\end{abstract}

Keywords: Uterine cervical cytodiagnosis, Morphologic features, Cancer nucleus, Kapur method, 8-direction contour tracing algorithm

\section{1: Introduction}

Cervical cancer shows fifth highest rate of incidence after breast cancer, gastric cancer, colorectal cancer, thyroid cancer among all malignant tumors that occurs to females. In addition, cervical cancer is the most prevalent cancer among female genital cancers. However, unlike other cancers, early detection and treatment of cervical cancer is possible by receiving regular cancer screening in early precancerous stage, the stage before the transition into cancer. Recently, due to development of medicine, various screening methods have increased the rate of early detection significantly[1]. Screening methods for cervical cancer includes pap smear, pelvic examination, speculum examination, HPV(human papillomavirus) DNA test and others. Among these screening methods, pap-smear is mainly used for screening at early precancerous stage[2]. During pap-smear screening, tissue samples are collected from various places around the cervix and stained to be examined under a microscope for any anomalies. This process produces fairly accurate screening results. However, in contrast to the high incidence rate of the cancer, the amount that can be screened by medical doctors and pathologists is limited. 
Recently, utilization of area expansion technique, fuzzy RBF network and Kapur method has been suggested for uterine cervical cell extraction and recognition. In cell extraction and recognition applied with area expansion technique and fuzzy RBF network, firstly expansion technique is applied in order to efficiently divide the cell area. Then hue value of the cell nucleus is extracted from the cell area, which is applied to fuzzy RBF network, classifying and recognizing normal cells and abnormal cells. However, this method classifies and recognizes normal cells and abnormal cells by applying hue value of cell nucleus on fuzzy RBF network without analyzing morphological features (structural changes, change in the hue of abnormal cells) of the cell. Therefore, this method cannot be applied to extraction and recognition of various uterine cervical cells such as large cell squamous cell carcinoma, intraepithelial carcinoma and others[3]. Traditional way of extracting and recognizing uterine cervical cell by Kapur method has a problem that the lowered accuracy of classifying normal cells and cancer cells caused by loss of cell nucleus information during pretreatment process of splitting background and cell area from pap-smear image, induces a higher false-negative rate and false-positive rate of cell differentiation[4].

Therefore, this paper intends to lower the false-negative rate and false-positive rate of cell screening and differentiation via assistive tool automated by study on the characteristics of uterine cervical cell nucleus used for pap-smear. Scaling of cell smear image used for examination by medical doctors and pathologists is 400-1000. In this paper, fuzzy membership function is designed and membership grade is extracted after analyzing characteristics of the morphological information in 400x magnified uterine cervical stratified squamous cell image of normal and cancerous cell nucleus. Then inference rules are applied to recognize normal and cancerous cell nucleus from uterine cervical cell image.

\section{2: Cell Nucleus Area Extraction Process}

The process for uterine cervical cell nucleus extraction is shown in Figure 1.

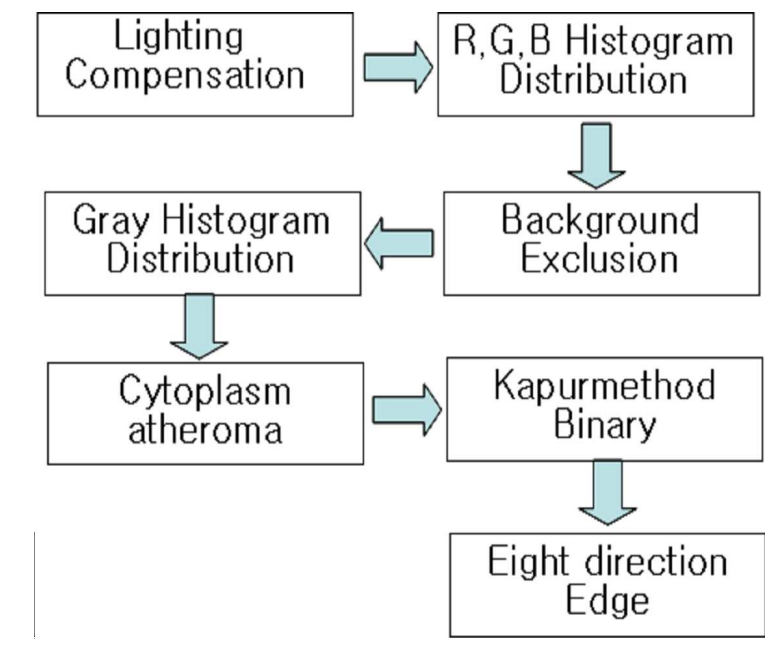

Figure 1. Nucleus Area Extraction Process 


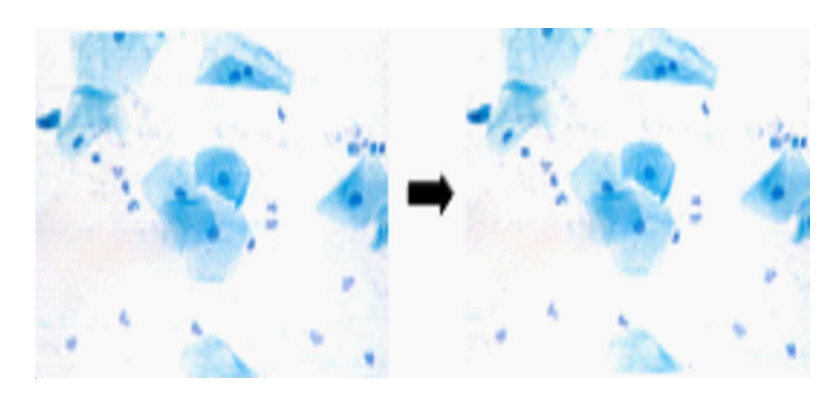

Figure 2. Images Before and After Correction of Color Information

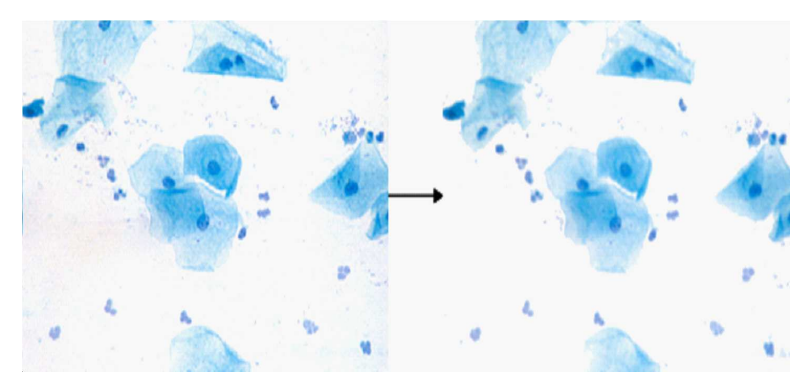

Figure 3. Images Before and After Background Removal by R, G, B Histogram Distribution

\section{1: Image Stabilization by Lighting Compensation}

In this paper, in order to select the candidate area for cell nucleus from uterine cervical cell image, we go through the process of restoring the color image damaged in the course of obtaining 400x magnified image. Lighting Compensation is applied to restore the image. Restored image is shown in Figure 2.

\section{2: Removal of Background Using the Histogram Distribution}

Background area is removed from the image which contains color information restored by lighting compensation, using the histogram distribution of R, G, B. Since the area with the highest density of R, G, B distribution is generally the background, after analysis of $\mathrm{R}, \mathrm{G}, \mathrm{B}$ histogram distribution, the area with top $10 \%$ distribution is regarded as the background area and is removed. Figure 3 shows the image of histogram distribution of R, G, B respectively and the image after the removal of background.

\section{3: Separation of Cytoplasm and Nucleus Using the Intensity Value}

After converting background area removed image to gray image, cytoplasm area and cell nucleus area is separated by analyzing histogram of image intensity and using the intensity value. Generally cytoplasm surrounding the cell nucleus has a lower intensity value than the cell nucleus. Therefore, in this paper, after analyzing the intensity histogram from the gray image, the area with top $5 \%$ highest intensity is regarded as the nucleus area, and area with lower intensity is regarded as the cytoplasm area surrounding the nucleus. Then the cell nucleus area is extracted by removing the cytoplasm area. Figure 4 shows the gray image and the distribution of intensity of the gray image. 


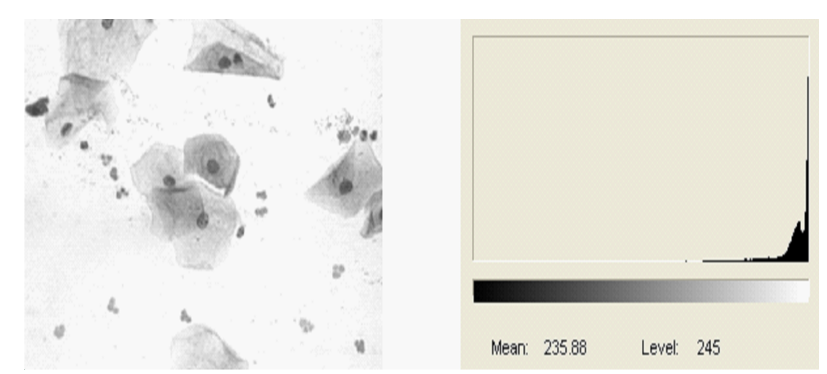

Figure 4. Gray Image and Its Histogram Distribution

\section{4: Cell Nucleus Binarization by Kapur Method}

In order to binarize the cytoplasm area-removed uterine cervical cell nucleus image, we applied Kapur method[4,5]. In Kapur method, after dividing acquired histogram of the image into two sections by a random gray level value $\mathrm{k}$ between $0^{\sim} 255$, by using equation (1) and (2) value of ' $k$ ' that makes the sum of $\mathrm{C}_{0}$ and $\mathrm{C}_{1}$ is set up as the threshold value of ' $\mathrm{k}$ '. Then the image is binarized based on the threshold value of ' $\mathrm{k}$ '. Entropy $\mathrm{C}_{0}$ of cell nucleus area and entropy $\mathrm{C}_{1}$ of non-interest area surrounding the cell nucleus is shown in equation (3) and (4). In these equations, $p_{i}$ indicates the $\mathrm{i}-$ th gray level value, and $\omega_{\mathrm{k}}$ indicates the cumulative rate from 0 to $\kappa$.

$$
\begin{gathered}
\text { class } C_{0}: \frac{p_{0}}{\omega_{k}} \frac{p_{1}}{\omega_{k}} \cdots \frac{p_{\mathrm{k}}}{\omega_{k}} \\
\text { class } C_{1}: \frac{p_{\mathrm{k}+1}}{L-\omega_{k}} \frac{p_{\mathrm{k}+2}}{L-\omega_{k}} \cdots \frac{p_{\mathrm{L}-1}}{L-\omega_{k}}
\end{gathered}
$$

The cumulative entropy of two classes is elucidated by equation (3) and (4).

$$
\begin{gathered}
\mathrm{H}\left(\mathrm{C}_{0}\right)=-\sum_{\mathrm{i}=0}^{\mathrm{k}} \frac{p_{\mathrm{i}}}{\omega_{\mathrm{k}}} \ln \frac{p_{\mathrm{i}}}{\omega_{\mathrm{k}}} \\
\mathrm{H}\left(\mathrm{C}_{1}\right)=-\sum_{\mathrm{i}=\mathrm{k}+1}^{\mathrm{L}-1} \frac{p_{\mathrm{i}}}{\mathrm{L}-\omega_{\mathrm{k}}} \ln \frac{p_{\mathrm{i}}}{\mathrm{L}-\omega_{\mathrm{k}}}
\end{gathered}
$$

$\psi(\mathrm{k})$, sum of two cumulative entropies, is shown in equation (5).

$$
\begin{gathered}
\psi(\mathrm{k})=\mathrm{H}\left(\mathrm{C}_{0}\right)+\mathrm{H}\left(\mathrm{C}_{1}\right) \\
=\ln \sum_{\mathrm{i}=0}^{\mathrm{k}}+\ln \sum_{\mathrm{i}=\mathrm{k}+1}^{\mathrm{L}-1}-\frac{\sum_{\mathrm{i}=0}^{\mathrm{k}} \mathrm{P}_{\mathrm{i}} \ln \mathrm{P}_{\mathrm{i}}}{\sum_{\mathrm{i}=\mathrm{o}}^{\mathrm{k}} \mathrm{P}_{\mathrm{i}}}-\frac{\sum_{\mathrm{i}=\mathrm{k}+1}^{\mathrm{L}-1} \mathrm{P}_{\mathrm{i}} \ln \mathrm{P}_{\mathrm{i}}}{\sum_{\mathrm{i}=\mathrm{k}+1}^{\mathrm{L}-1} \mathrm{P}_{\mathrm{i}}} \\
\mathrm{k}_{\text {opt }}=\operatorname{Max}(\psi(\mathrm{k}))
\end{gathered}
$$

At this point, $k_{\mathrm{opt}}$ means the value that makes the sum of entropy of two classes $\omega_{\mathrm{k}}$ and $\mathrm{L}-\omega_{\mathrm{k}}$ maximum. This image is binarized, with $\mathrm{k}_{\mathrm{opt}}$ fixed as the threshold value.

In an image applied with Kapur method, $3 \times 3$ mask is used to remove small noise. $3 \times 3$ mask scans the surrounding 8 directions. If there are more white pixels than black pixels, $3 \times 3$ mask removes the small noise existing in the image by replacing the center pixel with white. The structure of $3 \times 3$ mask is shown in Figure 5 . 


\begin{tabular}{|l|l|l|}
\hline 1 & 1 & 0 \\
\hline 1 & 1 & 0 \\
\hline 0 & 0 & 0 \\
\hline
\end{tabular}$\backsim$\begin{tabular}{|l|l|l|}
\hline 1 & 1 & 0 \\
\hline 1 & 0 & 0 \\
\hline 0 & 0 & 0 \\
\hline
\end{tabular}

Figure 5. 3 × 3 Mask Structure

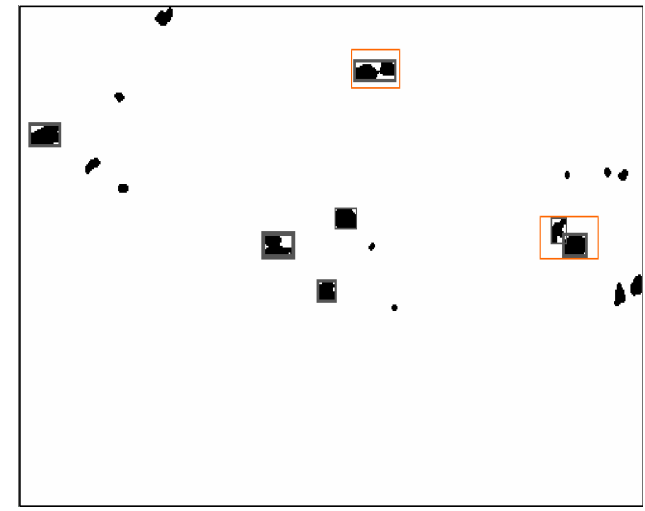

Figure 6. Nucleus Cluster Image

In the image with removed small noise, normal and cancerous nucleus cell is extracted by applying 8-direction contour algorithm. However, there are some cases in uterine cervical stratified squamous cell image where white blood cells and lymph cells are forming a cluster as shown in Figure 6.

In this paper, since we analyze the information of morphological characteristics of normal cell nucleus, we cannot extract the exact information of nuclear characteristics from area of cell nucleus forming a cluster with white blood cells and lymph cells. Therefore, in order to exclude the white blood cells and lymph cells from the area of interest, by using the average size of extracted cell nucleus, we decided that the area larger by more than $30 \%$ of average cell nucleus size is the clustered area. Then we removed the area with size smaller by $30 \%$ of the average cell nucleus size, which is the area of white blood cells and lymph cells, from the clustered area. The result of extracting normal cell nucleus and cancerous cell nucleus from binarized image by applying Kapur method and process of determining the threshold value in Kapur method is shown in Figure 7.

\section{3: Extraction of Cell Nucleus Characteristics}

Generally cancerous cell nucleus, which is larger than the normal cell nucleus, shows various morphology and possesses a distinct nucleoli. In addition, due to the increase of heterochromatin, chromatin of cancerous cell nucleus shows rough form. In fact, cytodiagnosis of cervical carcinoma is possible due to these features of cancerous cell nucleus[6]. In this paper, we classify and recognize normal and cancerous cell nucleus by using these following morphological characteristics of the cell nucleus; directional information of nuclear contour, area ratio and size of nucleus. Directional information is the information of nuclear contour roughness shown in numerical values. Area ratio indicates the degree of 


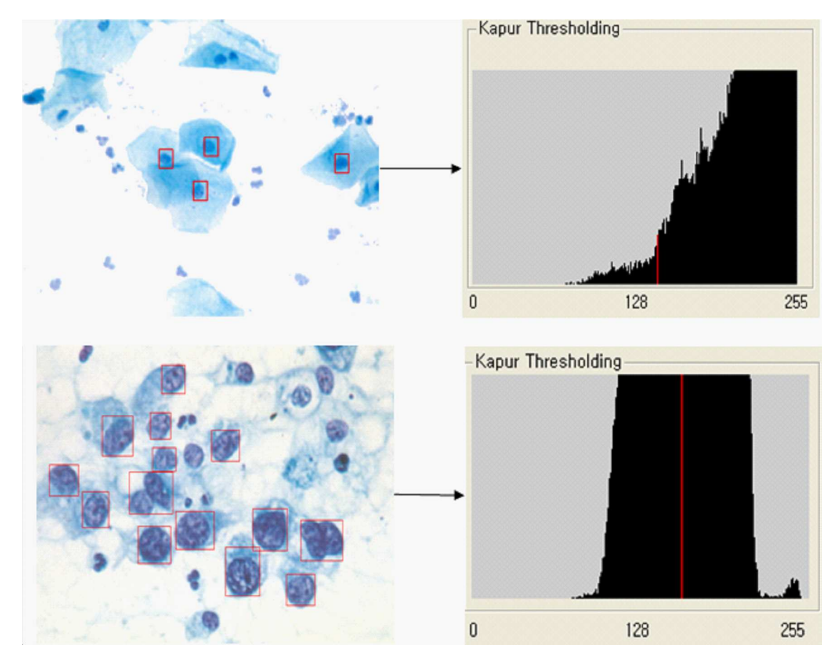

Figure 7. Extraction of Nucleus from Normal and Cancer Cell

darkness of the cell nucleus chromatin and the degree of how low the ratio of vacuole is, which is a structure that arises during the slow destruction of the cell. There is an inverse relationship between ratio of vacuole and area ratio. In addition, most cancerous cell nuclei show relatively larger size than the normal cell nuclei. Figure 8 shows the process of classifying cell nuclei based on their characteristics.

\section{1: Directional Information of Cell Nucleus}

In this paper, weights are applied to directions according to the progress direction of 8-directional contour tracing algorithm. Then direction value is obtained as the sum of all the differences between weights of former direction and next direction. This direction value is applied as the first feature for nucleus recognition. Each weight for eight directions are shown in Figure 9. In Figure 9, (a) shows the weights according to the direction and (b) shows a part of figures of DI, which is a sum of weights obtained from tracing the outline of each image. From (b), it can be seen that upper image with more rough outline shows a higher sum of weights despite the same perimeter as the lower image.

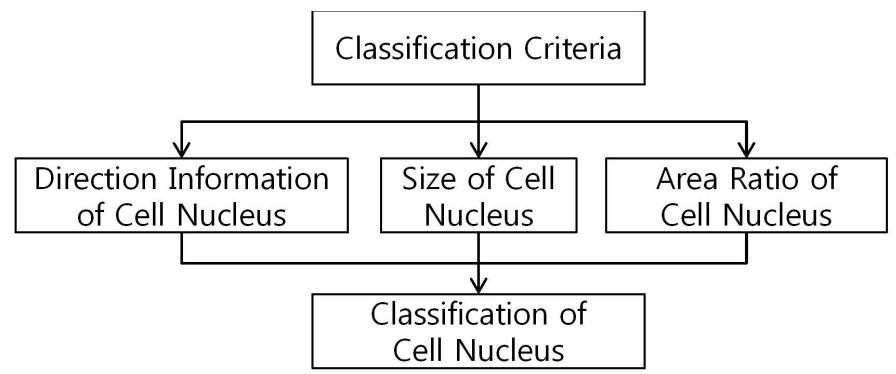

Figure 8. Nucleus Classification Process with Morphological Features 


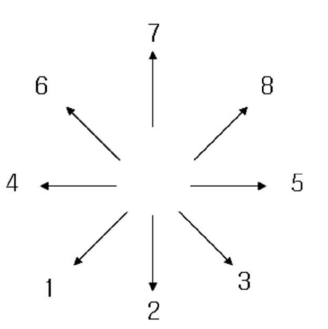

(a)

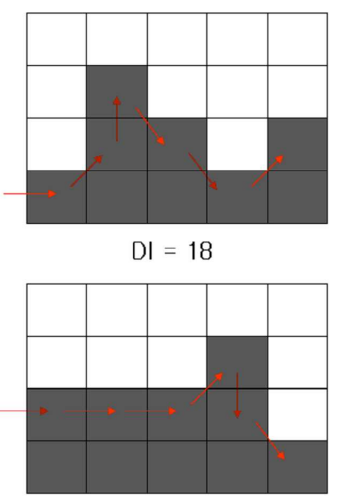

$\mathrm{DI}=15$

(b)

\section{Figure 9. (a) Weight by Direction (b) Sum of Weights from Images}

\section{2: Area ratio in Cell Nucleus}

Another feature of cancerous cell nucleus that differs from the normal cell nucleus is the vacuole that appears within the nucleus during the destruction of the nucleus. In order to apply this feature for recognition, we apply average binarization method on extracted single cell and calculate the ratio of vacuole within the cell nucleus to the total nucleus area as shown in equation (6). Normal cell and cancer cell with vacuole is compared in Figure 10 .

$$
\text { Cell Rate }=\text { Cell Size }- \text { Cell Hole }
$$

\section{3: Size of Nucleus}

Size of the extracted nucleus is also a significant feature. Normal nucleus is generally smaller than cancer nucleus. In addition, the ratio of nucleus to cytoplasm appears to be 1:8 1:9 in normal cells. However, in the case of nucleus in uterine cervical cancer cell, the possibility of the nucleus developing into dysplasia and metaplastic is over $90 \%$. Therefore, the size of the nucleus becomes larger due to the features of granules and heterochromatin. In addition, the cytoplasm also shows a rough morphology[2].

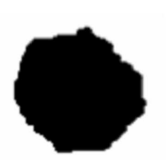

(a)

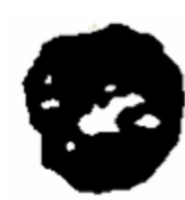

(b)

\section{Figure 10. (a) Normal Nucleus (b) Cancer Nucleus with Vacuole}




\section{4: Designing Membership Functions for Nucleus Recognition \& Nucleus Recognition by Fuzzy Reasoning Rules}

Membership function is designed in this paper to apply features of directional information, size and area ratio of the nucleus on fuzzy method during the process of recognizing extracted normal nucleus and cancer nucleus of uterine cervical. In this paper, membership functions are composed of three categories, Normal, Middle and Cancer. Normal is expressed as N, Middle as M and Cancer as C. Category M is set as the intersection of $\mathrm{N}$ section and $\mathrm{C}$ section. Calculated membership grade from each category is applied to output membership function to obtain fuzzy value, which is decided by Max_Min operation. Defuzzification is performed by applying center of gravity method on the result value from previous Max_Min operation. The nuclei of normal and cancer cells are finally recognized by calculating membership grades of normal and cancer cell nuclei through defuzzification. As shown in Figure 11, membership functions for directional feature of the nucleus possess membership section of $[0,10]$. N_D indicates the direction for section N, M_D indicates the direction for section $\mathrm{M}$ and $\mathrm{C} \_\mathrm{D}$ indicates the direction for section C. Membership section of directional features in the cell is distinguished by numerical value of directions obtained from progression direction of 8-direction contour tracing algorithm. Since normal nucleus has a form close to a circle, normal nucleus belongs to an N_D section of $[0,5]$ with a low numerical value of direction. In contrast, due to the increase of heterochromatin in the nucleus, cancer nucleus has a rough conformation which results in higher numerical value of direction. Therefore, cancer cell nucleus belongs to C_D section with high membership section of up to $[5,10]$. Since process of cell division and growth can be fast according to the menstruation cycle, even the surface of normal nucleus can become rough. In this case, M_D section is defined as the $[4,6]$ section which is an intermediate section of N_D, nuclear section of normal cells and C_D, nuclear section of cancer cells.

In Figure 12, L2, a section of membership function for the size of the nucleus, is a section of $[0,20]$. N_S indicates the size for Normal section, M_S indicates the size for intermediate section and C_S indicates the size for Cancer section. Normal nuclei share a regular size. Therefore, normal nucleus belongs to the N_S section of $[0,10]$ which implicates the small variance in size. In contrast, size of cancer nuclei is irregular and cancer nuclei shows various sizes. Thus, in the case of cancer nuclei, they belong to C_S section of $[10,20]$, implicating the large variance in their sizes. However, in some cases during the process of cell division and growth, observed size of normal nucleus was similar to that of cancer nucleus. In this case, an intermediate section of N_S and C_D were defined as M_S, a section of $[8,12]$.

In Figure 13, L3, a section of membership function for area ratio of nucleus, is a section of $[0,15]$. N_R is a area ratio for Normal section, M_R is a area ratio for intermediate section and $\mathrm{C} \_\mathrm{R}$ is a area ratio for Cancer section. In normal cells, the ratio of vacuole is very low

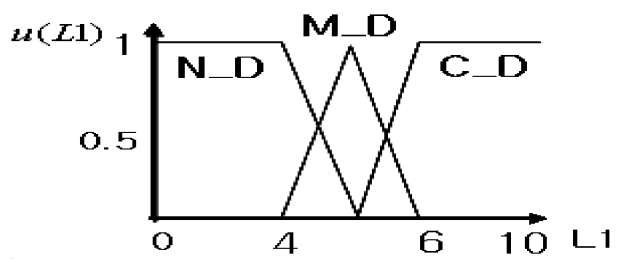

Figure 11. Membership Functions by Directional Information 


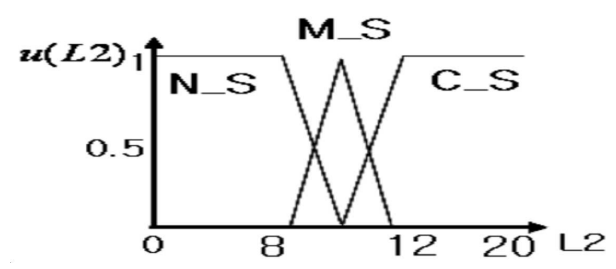

Figure 12. Membership Functions by the Size of Nucleus

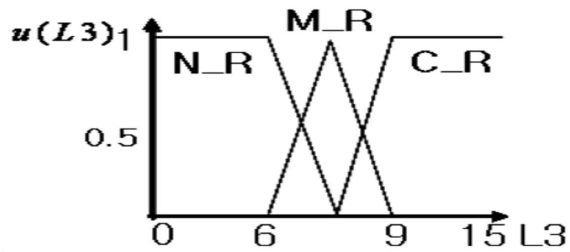

Figure 13. Membership Functions by Nucleus Area Ratio

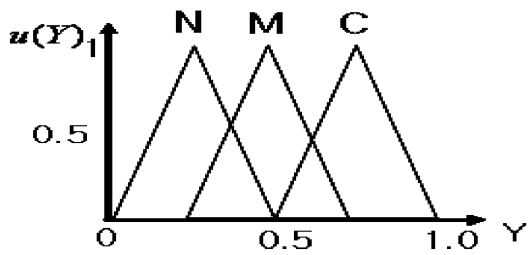

Figure 14. Output Membership Functions

since vacuole is hardly formed during the nucleus destruction. Therefore, section $[0,7.5]$ is a N_R membership section. However, cancer nucleus have a high membership section of $[7.5,15]$ for C_R due to the high ratio of vacuole. However, vacuole can be appear in normal cells due to aging phenomenon. For this case, M_R section is newly defined as section of $[6,9]$ which is an intermediate section of N-R and C_R. Membership functions for area ratio of nucleus is shown in Figure 13.

In this paper, membership grades calculated from membership function according to directional information, size and area ratio of the nucleus is decided by the following reasoning rules. Firstly, membership grade for directional information of the nucleus is $\mu\left(L_{1}\right)$. membership grade for nuclear size is $\mu\left(L_{2}\right)$. Finally, membership grade for area ratio of nucleus is $\mu\left(L_{3}\right)$. Figure 14 shows output membership function achieved from the input of each membership function. In this paper, reasoning rules applies directional information of the nucleus for the recognition of uterine cervical cell nucleus. Therefore, reasoning rules is divided into three cases; when directional information belongs to N_D section of normal cells or C_D section of cancer cells or M_D section, an intersection of normal nucleus and cancer nucleus. The results for three membership functions are indicated as Y, and reasoning is performed as shown below.

(1) When directional information of the nucleus belongs to Normal section

If L1 is N_D and L2 is N_S and L3 is N_R then Y is N

if $\mathrm{L} 1$ is N_D and L2 is N_S and L3 is C_R then $\mathrm{Y}$ is N

if L1 is N_D and L2 is N_S and L3 is M_R then Y is N 
if $\mathrm{L} 1$ is $\mathrm{N} \_\mathrm{D}$ and $\mathrm{L} 2$ is $\mathrm{C} \_\mathrm{S}$ and $\mathrm{L} 3$ is $\mathrm{N} \_\mathrm{R}$ then $\mathrm{Y}$ is $\mathrm{N}$ if L1 is N_D and L2 is C_S and L3 is M_R then Y is N If L1 is N_D and L2 is M_S and L3 is N_R then Y is M if $\mathrm{L} 1$ is N_D and L2 is M_S and L3 is C_R then $\mathrm{Y}$ is M if L1 is N_D and L2 is M_S and L3 is M_R then Y is M

(2) When directional information of the nucleus belongs to Cancer section

if $\mathrm{L} 1$ is $\mathrm{C} \_\mathrm{D}$ and L2 is N_S and L3 is N_R then Y is N if L1 is C_D and L2 is N_S and L3 is C_R then Y is C if $\mathrm{L} 1$ is $\mathrm{C} \_\mathrm{D}$ and L2 is C_S and L3 is N_R then Y is C if L1 is C_D and L2 is N_S and L3 is M_R then Y is C if $\mathrm{L} 1$ is $\mathrm{C} \_\mathrm{D}$ and $\mathrm{L} 2$ is $\mathrm{C} \_\mathrm{S}$ and $\mathrm{L} 3$ is M_R then $\mathrm{Y}$ is $\mathrm{C}$ if $\mathrm{L} 1$ is $\mathrm{C} \_\mathrm{D}$ and $\mathrm{L} 2$ is M_S and L3 is N_R then $\mathrm{Y}$ is M if $\mathrm{L} 1$ is $\mathrm{C} \_\mathrm{D}$ and $\mathrm{L} 2$ is $\mathrm{M} \_\mathrm{S}$ and $\mathrm{L} 3$ is $\mathrm{C} \_\mathrm{R}$ then $\mathrm{Y}$ is $\mathrm{M}$ if $\mathrm{L} 1$ is $\mathrm{C} \_\mathrm{D}$ and $\mathrm{L} 2$ is M_S and L3 is M_R then $\mathrm{Y}$ is $\mathrm{M}$

(3) When directional information of the nucleus belongs to Middle section

if L1 is M_D and L2 is N_S and L3 is N_R then Y is N if L1 is M_D and L2 is N_S and L3 is C_R then Y is N if $\mathrm{L} 1$ is $\mathrm{M} \_\mathrm{D}$ and L2 is $\mathrm{C} \_\mathrm{S}$ and $\mathrm{L} 3$ is $\mathrm{N} \_\mathrm{R}$ then $\mathrm{Y}$ is $\mathrm{C}$ if $\mathrm{L} 1$ is M_D and L2 is N_S and L3 is M_R then $Y$ is M if $\mathrm{L} 1$ is M_D and L2 is C_S and L3 is M_R then $\mathrm{Y}$ is M if $\mathrm{L} 1$ is M_D and L2 is M_S and L3 is N_R then $Y$ is M if $\mathrm{L} 1$ is M_D and L2 is M_S and L3 is C_R then $\mathrm{Y}$ is $\mathrm{M}$ if $L 1$ is M_D and L2 is M_S and L3 is M_R then $Y$ is $M$

For example, if L1, directional information of the nucleus, belongs to N_D section, L2, nuclear size, belongs to N_S and L3, area ratio of nucleus, belongs to N_D section, result is decided as the $\mathrm{N}$ section. As another example, if $\mathrm{L} 1$, directional information of the nucleus, belongs to C_D section, L2, nuclear size, belongs to C_S section and L3, area ratio of nucleus, belongs to N_R section, the result $\mathrm{Y}$ is decided as $\mathrm{C}$ section. Method for deciding membership section, which is obtained from applying reasoning rules, by Max_Min composite operation is shown in equation (7).

$$
\mu_{\mathrm{R}}\left(\mathrm{N}_{\mathrm{m}}, \mathrm{C}_{\mathrm{m}}, \mathrm{M}_{\mathrm{m}}, \mathrm{Y}_{\mathrm{m}}\right)=\max ?\left(\min \left(\mu_{\mathrm{N}}\left(\mathrm{N}_{\mathrm{i}}\right), \mu_{\mathrm{C}}\left(\mathrm{C}_{\mathrm{i}}\right), \mu_{\mathrm{M}}\left(\mathrm{M}_{\mathrm{i}}\right), \mu_{\mathrm{Y}}\left(\mathrm{Y}_{\mathrm{i}}\right)\right)\right)
$$

Among the results decided by Max_Min operation, the result with the highest membership grade is searched. The result value is defuzzified by applying center of gravity method. The calculation process for defuzzification is shown in equation (8)[7].

$$
\mathrm{y}^{*}=\frac{\sum \mu\left(\mathrm{y}_{\mathrm{i}}\right) \mathrm{x}_{\mathrm{i}}}{\sum \mu\left(\mathrm{y}_{\mathrm{i}}\right)}
$$

In equation (8), $\mathrm{y}^{*}$ indicates the output value of defuzzification. Table 1 shows final decision criteria for normal nucleus and cancer nucleus using value derived by defuzzification of fuzzy value obtained previously. 


\section{Table 1. Final Decision Criteria}

\begin{tabular}{|c|c|}
\hline Final decision & Decision criteria \\
\hline Normal nucleus & $0.0<=\mathrm{y}^{*}<=0.5$ \\
\hline Cancer nucleus & $0.5<\mathrm{y}^{*}<=1.0$ \\
\hline
\end{tabular}

\section{5: Methods and Results}

Experiment environment was implemented with Visual $\mathrm{C}++6.0$ on IBM compatible Pentium-IV PC. The experiment was performed on 12 normal uterine cervical stratified cell images and 20 cancerous uterine cervical stratified cell images with 400x magnification and 640x480 size. 59 normal nuclei were extracted from total 61 normal nuclei in normal uterine cervical cell images and 131 cancer nuclei were extracted from total 138 cancer nuclei in cancer cell images. Table 2 shows the nucleus recognition results for normal cell images and Table 3 shows the nucleus recognition result for cancer cell images. Table 4 shows nucleus recognition result for overall uterine cervical cell images. Figure 15 is the screen shot that shows extraction and recognition of uterine cervical cells proposed in this paper.

In the experimental results, there were cases when recognition of normal nucleus of stratified cell and superficial squamous cell failed.

Table 4.

In the case of nucleus with intermediate type, due to the fast cell division and growth according to menstruation cycle, the cell surface was rough and the size increase. Vacuole was also formed within the nuclear surface. Therefore, this type of nucleus was recognized as a cancer nucleus when decided by applying membership function for directional infor-

Table 2. Recognition Results for Normal Cells

\begin{tabular}{|c|c|c|}
\hline Normal cell image & \# of recognized nuclei & Total \# of nuclei \\
\hline stratified squamous cell & 12 & 12 \\
\hline stratified stratum intermedium cell & 18 & 19 \\
\hline columnar epithelium cell & 13 & 13 \\
\hline superficial squamous cell & 16 & 17 \\
\hline
\end{tabular}

Table 3. Recognition Results for Cancer Cells

\begin{tabular}{|c|c|c|}
\hline Cancer cell image & \# of recognized nuclei & Total \# of nuclei \\
\hline Keratinous cell carcinoma & 12 & 12 \\
\hline Non-keratinized cell carcinoma & 17 & 19 \\
\hline Metaplastic cell carcinoma & 13 & 13 \\
\hline Large cell squamous cell carcinoma & 15 & 17 \\
\hline Small cell squamous cell carcinoma & 6 & 6 \\
\hline Minimal infiltrative cell carcinoma & 16 & 16 \\
\hline Clear cell gland cell carcinoma & 10 & 10 \\
\hline Intraepithelial carcinoma & 24 & 24 \\
\hline Dysplasia cell carcinoma & 18 & 21 \\
\hline
\end{tabular}


Table 4. Results of Recognition and Extraction by the Proposed Algorithm

\begin{tabular}{|c|c|c|}
\hline Final Dicision & Total \# of recognized cells & Total \# of cells \\
\hline Normal nucleus & 59 & 61 \\
\hline Cancer nucleus & 131 & 138 \\
\hline
\end{tabular}

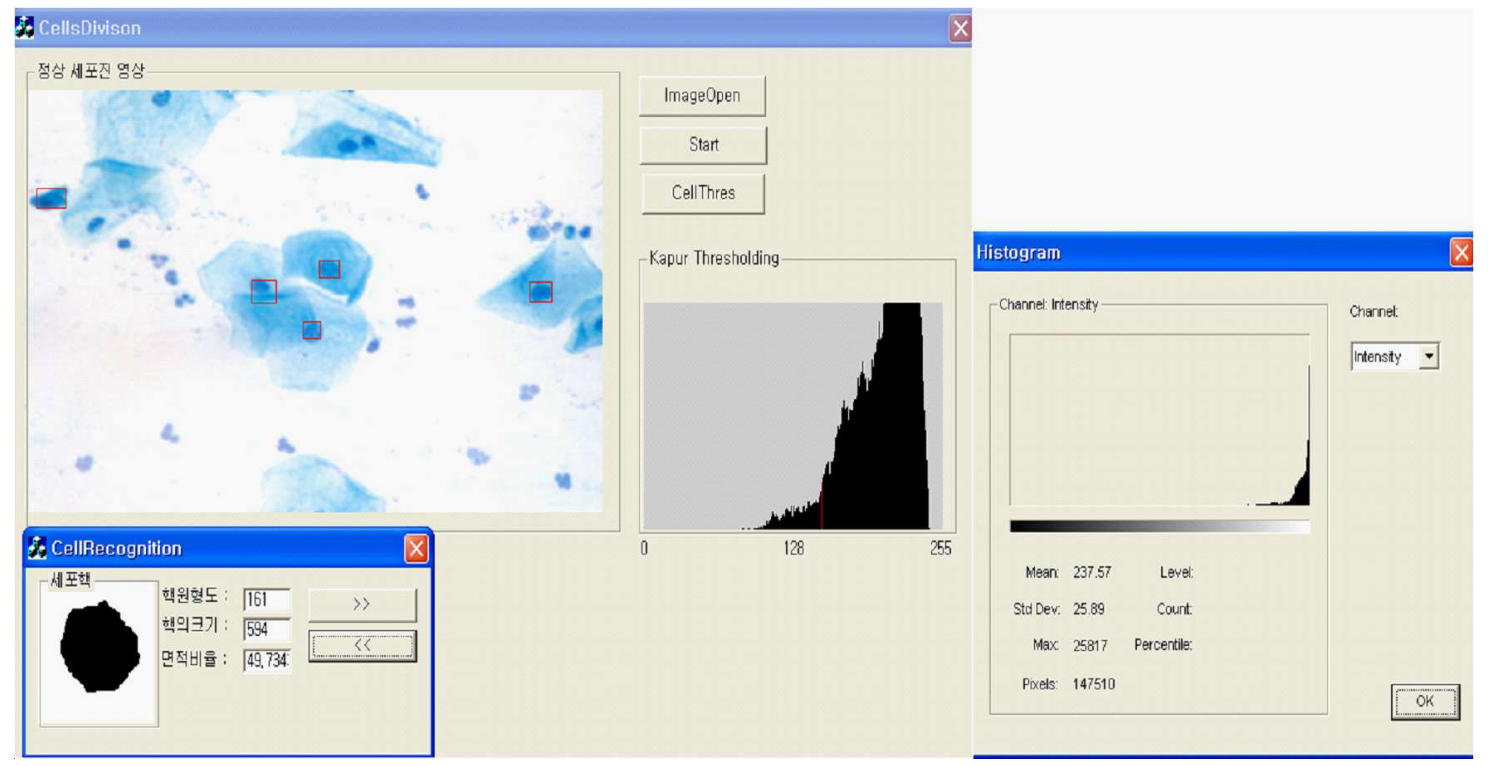

Figure 15. Screen Shot of the Extraction and Recognition of Uterine Cervical PapSmears

mation, size and area ratio of the nucleus. In the case of cancer nuclei, non-keratinized cell carcinoma, large cell squamous cell carcinoma and dysplasia cell carcinoma were sometimes recognized as normal nuclei. In the case of non-keratinized cell carcinoma, vacuole does form. However, the nuclear surface does not change and the cell size is also similar to that of a normal cell. Therefore process failed to recognize non-keratinized cell carcinoma as a cancer cell. Only the cell size changed in large cell squamous cell carcinoma. In the case of dysplasia cell carcinoma, since it is a cancer cell derived from inflammation, the nuclear surface becoming rough was the only change. Therefore, in these cases, the cancer cells were recognized as normal cells when fuzzy reasoning method was applied.

\section{6: Conclusion}

In this paper, in order to extract the nucleus from uterine cervical pap-smear image used for pap smear, we applied Lighting Compensation to compensate the image. Then we removed the background using the histogram distribution of R, G, B area in the image to distinguish the nucleus area from the background area. After converting the image with removed background to gray image, we separated the nucleus and cytoplasm using intensity value of histogram. Kapur method was applied to calculate probabilities of cumulative entropy, which was used to binarize the image. Small noises were eliminated from the binarized image applied with Kapur method by applying 3x3 mask, a method comparing 
the center and the peripheral pixels of nucleus area. After removing the small noises, the nucleus was finally extracted by 8-direction contour tracing algorithm. As a process of classifying and recognizing extracted nuclei into normal or cancer nuclei, features of direction information of cell contour, size of nucleus and area ratio was separately obtained. Membership grades were then obtained by applying each features into their respective fuzzy membership function. Finally, fuzzy reasoning rule was applied to recognize normal nucleus and cancer nucleus from the uterine cervical pap-smear image. There are approximately 100 features used for classifying and distinguishing cells. However, selecting the features of cancer cells is a difficult process and it takes a long time to extract the features of the cancer cells. Among the various features, 3 features, size, area and direction was applied to fuzzy reasoning rules in this paper. The result showed that 190 nuclei among 199 normal and cancer nuclei were correctly recognized and 9 nuclei were incorrectly recognized.

Further research will analyze the features of morphological alteration of nucleus during menstruation cycle of females and developmental process of uterine cervical cell nucleus. The research will further proceed to studying methods to recognize not only the cell nuclei of stratified squamous cell, but also other various types of cell nuclei of uterocervical canal and surface of uterus.

\section{References}

[1] Park Kwan-Kyu, Kwon Sun-Young, Chang Eun-Sook, Jegal Moon-Young, Han SangMi, Cha Soon-Do and Han In-Sook, "Early Detection of Metastasis by Immunohistochemistry in Uterine Cervical Carcinoma", The Korean Journal of Pathology, vol.35, no.5, (2001), pp.391-400.

[2] Sung Kyung Un, Chan Mo Park, Hwa Choon Park, So Young Yoon, Min Sun Cho, Soo Yeon Cho and Sung Sook Kim, "A Study on an Automatic Detection of Nucleus from Pap-smears by Image Processing", Korean journal of cytopathology, vol. 5, no. 1, (1994), pp.15-22.

[3] K. B. Kim, D. H. Song, Y. W. Woo, " Nucleus Segmentation and Recognition of Uterine Cervical Pap-Smears", Lecture Notes in Artificial Intelligence, vol. 4482, (2007), pp.153160.

[4] K. B. Kim, "Detection and Recognition of Uterine Cervical Carcinoma Cells in Pap Smear Using Kapur Method and Morphological Features", The journal of the Korea Institute of Maritime Information \& Communication Sciences , vol.11, no.10, (2007), pp.1992-1998.

[5] J.N. Kapur, P.K. Sahoo, A.K.C. Wong, "A New Method for Gray Level Picture Thresholding Using the Entropy of the Histogram", Computer Vision, Graphics, and Image Processing, vol.29, (1985), pp.273-285.

[6] M. S. Kim and S. W. Juhng, "mRNA is Synthesized at the Phase between Euchromatin and Heterochromatin: Proposal of a Phase Theory", Korean Journal of Pathology, vol.35, (2001), pp.93-97.

[7] R. Babuska, Fuzzy Modeling For Control, Kluwer Academic Publishers, 1998. 


\section{Authors}

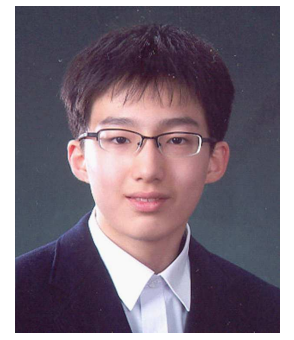

Hyunjun Woo is undergraduate student in college of medicine, Seoul National University, Seoul, Korea. His research interests include medicine, medical information.

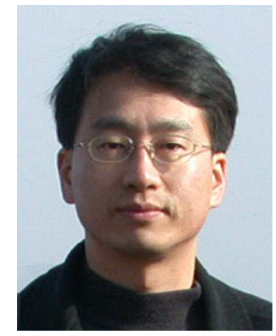

Young Woon Woo received the B.S. degree, M.S. degree and Ph.D. degree in electronic engineering from Yonsei University, Seoul, Korea in 1989, 1991 and 1997, respectively. Since 1997, he has been a professor in Department of Multimedia Eng., Dong-Eui University, Busan, Korea. His research interests are in the area of artificial intelligence, image processing, pattern recognition and medical information.

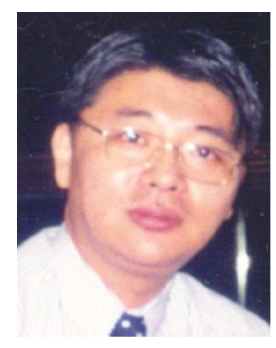

Kwang-Baek Kim(corresponding author) received his M.S. and the Ph.D. degrees in Department of Computer Science from Pusan National University, Busan, Korea, in 1993 and 1999, respectively. From 1997 to present, he is a professor, Department of Computer Engineering, and Silla University in Korea. He is currently an associate editor for Journal of The Korea Society of Computer and Information, and The Open Artificial Intelligence Journal (USA). His research interests include fuzzy neural network and application, bioinformatics and image processing. 